\title{
BMJ Open Cardiovascular imaging of women and men visiting the outpatient clinic with chest pain or discomfort: design and rationale of the ARGUS Study
}

Floor Groepenhoff (D) , ${ }^{1,2}$ Anouk L M Eikendal, ${ }^{2}$ Sophie Heleen Bots (D) , ${ }^{2}$ Anne-Mar van Ommen, ${ }^{2}$ L M Overmars, ${ }^{1}$ Daniek Kapteijn, ${ }^{2}$ Gerard Pasterkamp, ${ }^{1}$ Johan H C Reiber, ${ }^{3}$ David Hautemann, ${ }^{3}$ Roxana Menken, ${ }^{4}$ Marianne E Wittekoek, ${ }^{5}$ Leonard Hofstra, ${ }^{4} \mathrm{~N}$ Charlotte Onland-Moret, ${ }^{6}$ Saskia Haitjema, ${ }^{1}$ Imo Hoefer, ${ }^{1}$ Tim Leiner, ${ }^{7}$ Hester M den Ruijter ${ }^{2}$

To cite: Groepenhoff $F$, Eikendal ALM, Bots SH, et al. Cardiovascular imaging of women and men visiting the outpatient clinic with chest pain or discomfort: design and rationale of the ARGUS Study. BMJ Open 2020;10:e040712. doi:10.1136/ bmjopen-2020-040712

- Prepublication history and supplemental material for this paper are available online. To view these files, please visit the journal online (http://dx.doi. org/10.1136/bmjopen-2020040712).

Received 20 May 2020 Revised 17 0ctober 2020 Accepted 09 November 2020

Check for updates

(C) Author(s) (or their employer(s)) 2020. Re-use permitted under CC BY-NC. No commercial re-use. See rights and permissions. Published by BMJ.

For numbered affiliations see end of article.

\section{Correspondence to}

Professor Dr. Ir. Hester M den Ruijter;

h.m.denRuijter-2@umcutrecht. $\mathrm{nl}$

\section{ABSTRACT}

Introduction Chest pain or discomfort affects $20 \%-40 \%$ of the general population over the course of their life and may be a symptom of myocardial ischaemia. For the diagnosis of obstructive macrovascular coronary artery disease (CAD), algorithms have been developed; however, these do not exclude microvascular angina. This may lead to false reassurance of symptomatic patients, mainly women, with functionally significant, yet non-obstructive coronary vascular disease. Therefore, this study aims to estimate the prevalence of both macrovascular and microvascular coronary vascular disease in women and men presenting with chest pain or discomfort, and to subsequently develop a decision-support tool to aid cardiologists in referral to cardiovascular imaging for both macrovascular and microvascular CAD evaluation.

Methods and analysis Women and men with chest pain or discomfort, aged 45 years and older, without a history of cardiovascular disease, who are referred to an outpatient cardiology clinic by their general practitioner are eligible for inclusion. Coronary CT angiography is used for anatomical imaging. Additionally, myocardial perfusion imaging by adenosine stress cardiac MRI is performed to detect functionally significant coronary vascular disease. Electronic health record data, collected during regular cardiac work-up, including medical history, cardiovascular risk factors, physical examination, echocardiography, (exercise) ECG and blood samples for standard cardiovascular biomarkers and research purposes, are obtained. Participants will be classified as positive or negative for coronary vascular disease based on all available data by expert panel consensus (a cardiovascular radiologist and two cardiologists). After completion of the clinical study, all collected data will be used to develop a decision support tool using predictive modelling and machine-learning techniques.

Ethics and dissemination The study protocol was approved by the Institutional Review Board of the University Medical Center Utrecht. Results will be disseminated through national and international conferences and in peer-reviewed journals in cardiovascular disease.

\section{Strengths and limitations of this study}

ARGUS is the first study to perform cardiovascular imaging in early stage of the regular care pathway of patients with non-acute chest pain or discomfort.

- Extensive cardiovascular imaging will be obtained in all patients, not only if clinically indicated.

- Possible referral bias from the general practitioner to outpatient cardiology clinics.

- Lack of invasive measurements to verify microvascular angina or detect coronary vasospasm.

Trial registration number Trialregister.nl Registry NL8702.

\section{INTRODUCTION}

Chest pain or discomfort affects $20 \%-40 \%$ of the general population over the span of their life and may be a symptom of myocardial ischaemia. ${ }^{1}$ Approximately $40 \%$ of these patients are referred to an outpatient cardiology clinic for cardiac work-up to evaluate if the underlying cause is indeed myocardial ischaemia. ${ }^{1}$ However, distinguishing a possibly life-threatening cardiac aetiology from a non-cardiac cause in a short period of time remains a challenge.

Current clinical diagnostic decisionsupport tools have been solely developed to aid the cardiologist in diagnosing or excluding myocardial ischaemia due to obstructive, that is, anatomical, coronary artery disease (CAD).$^{2-5}$ Coronary CT angiography (CCTA) is well established to identify (non-)obstructive burden of anatomical coronary atherosclerotic disease and provides important information regarding CAD risk, also in women. ${ }^{6}$ However, invasive imaging 
studies in symptomatic patients reveal a high prevalence $(63 \%-71 \%)$ of anatomically non-obstructive, yet functionally significant, CAD. ${ }^{7-9}$ Non-obstructive CAD has been considered a benign condition for years, but recently appeared to be a significant cause of myocardial ischaemia due to observations of coronary vasospasms and microvascular angina (MVA). ${ }^{10}{ }^{11}$ Furthermore, the prognosis for both sexes regarding non-obstructive CAD appears to be as poor as that of patients with obstructive CAD. ${ }^{12} 13$

Despite increased awareness of the importance of coronary function testing for MVA and coronary spasms, its assessment remains a challenge carrying non-negligible risks and costs. However, recently several non-invasive imaging techniques have provided surrogate measures of the coronary microcirculation. ${ }^{14}$ Yet, the used imaging modality highly depends on local expertise, availability and costs. ${ }^{15}$ Cardiac MRI (CMRI) is emerging as a noninvasive technique with high spatial resolution to assess myocardial perfusion, that is, functional CAD, without the need for ionising radiation. ${ }^{16}{ }^{17}$ In fact, Knuuti et al confirmed CMRI to perform well for both anatomical and functional CAD. ${ }^{18}$ Therefore, the 2019 European Society of Cardiology (ESC) recommendations mention stress CMRI as an option that may be considered in patients with suspected coronary vascular disease. ${ }^{9}$

Hence, to add to the existing knowledge regarding obstructive $\mathrm{CAD}$ in symptomatic patients clinically referred for imaging, this study will assess the prevalence of both anatomical obstructive (using CCTA) and (non-) obstructive, yet significant, functional CAD (using stress CMRI) in all patients with chest pain or discomfort visiting the outpatient cardiology clinics. Additionally, we aim to develop a decision-support tool that aids cardiologists in referral for either CCTA and/or stress CMRI and study whether extensive phenotyping could improve long-term outcomes.

\section{METHODS AND ANALYSIS \\ Study design}

ARGUS is a single centre, prospective cohort study performed at the University Medical Center (UMC) Utrecht in the Netherlands. The study is actively recruiting women and men who visit cardiac outpatient clinics in Utrecht with chest pain or discomfort as the main reason for their visit. Currently, the Cardiology Centers of the Netherlands (CCN), location Utrecht, and HeartLife Klinieken Utrecht are referring centres for participant recruitment. The study procedures are conducted at the radiology department of the UMC Utrecht.

\section{Study population}

The study population comprises women and men aged 45 years and older with chest pain or discomfort as primary report who are referred by their general practitioner to the participating outpatient cardiology clinics. Exclusion criteria are listed in box 1 and include cardiac history and
Box 1 Inclusion and exclusion criteria for the ARGUS Study

Inclusion criteria:

- Age $\geq 45$ years.

- Willing and able to provide written informed consent.

- Chest pain or chest discomfort as main reason for visit.

- Willing and able to undergo complete study protocol.

Exclusion criteria

- Any cardiac history.*

- Contraindications to CCTA prior to inclusion.

- Contraindications to CMRI prior to inclusion.

- Contraindications to adenosine (second-degree or third-degree AV block, COPD or asthma with evidence of bronchospasm).

Inclusion and exclusion criteria are assessed by both the cardiologist and research physician before enrolment through patient records and direct contact with the participant on inclusion.

*Cardiac history includes: congenital heart disease, former cardiac procedures (angioplasty, myocardial infarction, bypass surgery, heart valve surgery or intervention, implantable cardiac defibrillator and/or cardiac resynchronisation therapy, radiofrequency ablation, left ventricular assist device, heart transplantation and pacemaker implantation). $\mathrm{AV}$, atrioventricular; CCTA, coronary CT angiography; CMRI, cardiac MRI; COPD, chronic obstructive pulmonary disease.

contraindications to undergo any of the study procedures. If CCTA or stress CMRI is contraindicated after part of the study protocol is already completed, the participant will not be excluded and collected data will be used in the study.

\section{Participant recruitment}

The treating cardiologist at the referring centre invites eligible patients to participate in the study at the end of the regular care visit. Participants that show interest in the study are contacted by the executive researcher. If these individuals provide informed consent, meet all inclusion criteria and none of the exclusion criteria, they are enrolled and undergo the study procedure. The study procedure consists of a standardised questionnaire, venous blood collection for measurement of known cardiovascular biomarkers and for storage in a biobank for later biomarker studies, as well as imaging by both CCTA and stress CMRI. If participants are already scheduled to undergo a clinically indicated CCTA, these results are obtained for study purposes and the CCTA would not be performed as part of the study procedure. The workflow of the participant recruitment and imaging protocol for both participants with and without clinically indicated CCTA is visualised in figure 1.

\section{Sample size considerations}

Prior studies found CCTA-defined anatomical CAD to be present in $11.9 \%$, and CMRI-defined myocardial ischaemia in about $12.6 \%$ in low-risk to intermediate-risk patients with chest pain. ${ }^{19}$ Since we expect our population to be mostly low-risk patients, we suspect the CAD prevalence to be at most $11 \%$. Based on our sample size calculation, a number of 400 would be sufficient to estimate a 


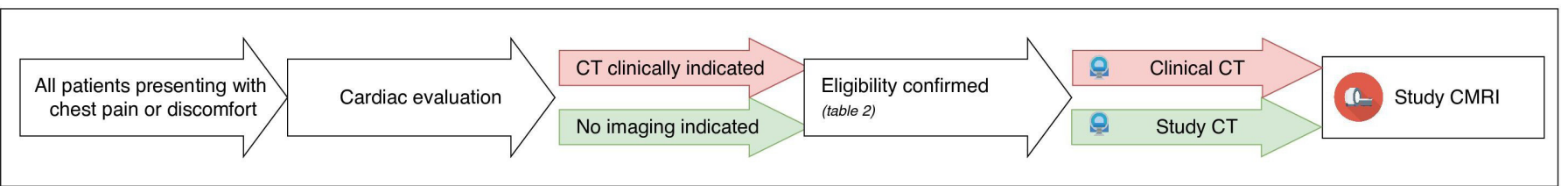

Figure 1 Workflow of patient recruitment and imaging protocol depending on regular clinical care path. CMRI, cardiac MRI.

prevalence of $11 \%$ with a precision of $3 \%$, using a $95 \%$ CI (https://epitools.ausvet.com.au/oneproportion). Thus, the ARGUS Study will run for 3 years, in which we expect to enrol 400 subjects and perform 400 CCTA and accompanying stress CMRI scans.

\section{Regular care work-up at the outpatient clinic}

Data collected during regular care include medical history, physical examination, ECG, laboratory blood measurements, exercise testing and transthoracic echocardiography. Comorbidities and medication use are registered using a standardised questionnaire. All regular care measurements (including information on follow-up visits and performed external procedures) are recorded in the local electronic health records of the outpatient clinics and extracted for analysis in the study.

\section{Study protocol}

Case report form

After obtaining informed consent, a study physician or trained research nurse fills out an extensive case report form with the participant, including information on symptoms (ie, chest pain or chest discomfort specific characteristics such as location, character, duration, provoking or alleviating factors such as relation to exercise, emotional stress, medication use, or other symptoms such as dyspnoea, palpitations or fatigue), cardiovascular risk factors, medical history, family cardiovascular history (first-degree relatives with cardiovascular events), level of education and medication use. Additionally, pregnancy history and menopausal status are recorded for women.

\section{Blood sampling}

A venous blood sample $(75 \mathrm{~mL})$ from a peripheral vein is collected and processed according to our existing biobank protocol. ${ }^{20} \mathrm{In}$ short, $2.5 \mathrm{~mL}$ EDTA plasma tube is analysed by the clinical chemistry department of the UMC Utrecht by an Abbott CELL-DYN Sapphire to obtain complete blood cell counts and additional raw blood cell characteristics. Furthermore, EDTA, citrate and sodium-heparin plasma and peripheral blood mononuclear cells isolated from EDTA blood are aliquoted and frozen at $-80^{\circ} \mathrm{C}$ within 2 hours after the sample was collected. All samples are stored long term at UMC Utrecht Central BioBank for future studies, except for 1 aliquot of $700 \mu \mathrm{L}$ which is transported to the Durrer Center for Cardiovascular Research (Netherlands Heart Institute, Amsterdam, the Netherlands). As previously described, standard cardiac biomarker levels (B-type natriuretic peptide (BNP) and high-sensitivity troponin-I (hs-TnI), Abbott Diagnostics,
Abbott Park, Illinois, USA) will be measured in plasma of all participants. ${ }^{20}$

\section{Cardiac and coronary CT angiography \\ Protocol}

Participants are asked to refrain from caffeine consumption for 4 hours and from food and nicotine for 2 hours prior to imaging. If the resting heart rate exceeds 60 beats/min, heart rate-lowering premedication can be prescribed based on clinical judgement of the treating cardiologist.

CCTA acquisitions will be performed using a 64-slice dual-layer spectral detector CT scanner (IQ on Spectral CT, Philips Healthcare, Best, the Netherlands) with $64 \times 0.625 \mathrm{~mm}$ collimation. In patients with a clinically scheduled CCTA in whom the scan could not be scheduled on the above-mentioned scanner, acquisitions will be performed according to local protocol, using either a 256-row multidetector scanner (Brilliance iCT, Philips Healthcare, Best, the Netherlands) or a 196-row CT scanner (SOMATOM Force, Siemens Healthcare Diagnostics). Detailed scan parameters and acquisition protocol specifications are listed in online supplemental appendix 1.

In participants with a heart rate above 60 beats $/ \mathrm{min}$ at time of the examination, intravenous beta-blocker will be administered in line with the Society of Cardiovascular CT guidelines (SCCT).$^{21}$ First, all participants will undergo coronary calcium scoring, which will be quantified by Agatston scores using commercially available software (HeartBeat CS, IntelliSpace Portal, Philips Healthcare, Best, the Netherlands).$^{21}{ }^{22}$ Acquisitions will be made using a prospectively ECG-triggered protocol at the $78 \%$ of the R-R interval at $120 \mathrm{kVp}$ and $40 \mathrm{mAs}$. All acquisitions will be performed according to the recent SCCT guidelines. ${ }^{21}$ All images will be analysed using a dedicated workstation (IntelliSpace Portal, Philips Healthcare, Best, the Netherlands). Subsequently, a CCTA scan will be acquired using the contrast agent $300 \mathrm{mg} \mathrm{I} / \mathrm{mL}$ Iopromide (Ultravist 300, Bayer Healthcare, Berlin, Germany) administered at a flow rate of $5.5 \mathrm{~mL} / \mathrm{s}(<80 \mathrm{~kg})$ or $6.0 \mathrm{~mL} / \mathrm{s}(\geq 80 \mathrm{~kg})$. Prior to the examination, sublingual nitroglycerin $0.4 \mathrm{mg}$ will be administered in all participants. After contrast injection, the intravenous line will be flushed using saline at the same flow rate. Seven seconds after the threshold of 200 Hounsfield units is reached in the descending aorta, the CCTA scan is acquired (prospectively ECG-triggered, $78 \%$ of the R-R interval at $120 \mathrm{kVp}$ and $150 \mathrm{mAs}$ ). 


\section{Analysis}

Following SCCT guidelines all images will be assessed on image quality, plaque characteristics and degree of stenosis by the local cardiovascular radiologists. ${ }^{21}$ To provide a standardised assessment of CCTA, the Coronary Artery Disease-Reporting and Data System (CADRADS) classification will be reported. ${ }^{23}$ The estimated radiation dose for the CCTA examination is $5.7 \mathrm{mSv}$.

\section{Adenosine stress perfusion CMRI \\ Protocol}

Stress CMRI will be performed on 1.5 or 3T scanners (Ingenia, Software release 5, Philips Healthcare, Best, the Netherlands) following a standardised protocol (online supplemental appendix 2). Twenty-fourhours before all participants will be asked to avoid caffeine, first, native T1-weighted images will be acquired. Afterwards, stress and rest myocardial perfusion imaging will be performed during the first pass of gadobutrol (Gadovist, Bayer, Germany). Vasodilator stress will be achieved by adenosine infusion at $140 \mu \mathrm{g} / \mathrm{kg} / \mathrm{min}$ for $4 \mathrm{~min}$. Rest perfusion imaging will be acquired at least $1 \mathrm{~min}$ after ending of adenosine infusion. Subsequently, cardiac function, post-contrast T1-weighted and viability imaging will be performed. The total duration will be approximately $50 \mathrm{~min}$.

\section{Image analysis}

All images will be analysed by the local supervising CMRI radiologists per regular clinical practice in accordance with the Society for Cardiovascular Magnetic Resonance (SCMR) guidelines. ${ }^{17}$ They will assess image quality and report data on native-contrast and post-contrast $\mathrm{T} 1$ values, quantitative functional analysis regarding end-diastolic and end-systolic volumes, ejection fraction, evidence of regional wall motion abnormalities (akinetic, hypokinetic or dyskinetic). Left ventricle strain imaging will be performed in long-axis cine images using Medis Suite MR software (Medis Medical Imaging Systems BV, Leiden, the Netherlands). Subsequently, analysis of significant, not significant or no defect in perfusion at rest and stress, presence of scar tissue and transmurality based on the American College of Cardiology/American Heart Association 17 segment model will be reported. ${ }^{24} \mathrm{~A}$ perfusion defect is scored as a significant perfusion defect if ischaemia is present in at least two segments and defined according to transmurality and persistence of the defect in accordance with the SCMR guidelines. ${ }^{17}$

\section{Definition of coronary vascular disease}

The primary aim of the study is to provide insight into the prevalence of anatomical and functional coronary vascular disease. Coronary vascular disease is defined as either stress CMRI-detected functional and/or CCTAdetected anatomical coronary vascular disease. Participants will be classified as positive or negative for any coronary vascular disease by expert panel consensus. The panel of experts comprises of a cardiovascular radiologist and two cardiologists. The panel reviews all available data to reach a consensus whether coronary vascular disease is likely to be the underlying cause of the chest pain or discomfort (figure 2). ESC guidelines for chronic coronary syndromes are followed. ${ }^{25}$ First, the panel assesses the symptoms and signs to classify type of angina according to the traditional classification of suspected anginal symptoms (typical, atypical or non-anginal chest pain). Second, the general condition, risk factors, comorbidities and basic testing results collected during regular care are interpreted by the panel. Based on these regular care data, the expert panel determines whether a cardiac cause is present (yes, no, possible) and, if so, whether they suspect the cause to be of macrovascular or microvascular or vasospastic origin. Following provision of the imaging results in combination with cardiac biomarker levels (BNP and hs-TnI), the expert panel re-evaluates their decision. The workflow of the data collection and subsequent diagnostic approach by the expert panel is visualised in figure 2 .

The imaging results for both functional and anatomical coronary vascular disease will be documented separately and provided to the expert panel as follows: CCTAdetected anatomical coronary vascular disease based on the CADRADS score assigned by the radiologist and stress CMRI-detected functional coronary vascular disease based on visual assessment of presence or absence of myocardial ischaemia by the radiologist (table 1$){ }^{23}$

\section{Long-term follow-up}

By linkage with regional (Julius General Practitioners' Network) and national registries (ie, Statistics Netherlands), all participants will be followed for consumption of medical care, occurrence of cardiac events, and allcause and cause-specific mortality. ${ }^{26} 27$

\section{Statistical analyses}

Descriptive data will be stratified by sex and presented as mean $\pm \mathrm{SD}$, or as medians with corresponding IQR if not normally distributed for continuous variables and numbers (with \%) for categorical variables. The prevalence of coronary vascular disease will be expressed as mean with a $95 \%$ CI. Multivariable logistic regression will be used to analyse the association of various determinants at baseline with prevalent coronary vascular disease and the association between various anatomical and functional parameters at baseline. All analyses will be adjusted for appropriate a priori selected confounders (age, sex, medical history, comorbidities and cardiovascular risk factors). For prognostic studies, Cox proportionalhazards regression analysis will be used. All analyses will be performed both in the whole study population as well as stratified by sex.

The collected data are part of the development of a decision-support tool. By means of machine-learning techniques, an algorithm will be trained to identify patterns between input data and outcome variables. Based on clinical data and extensive blood-based information as input 


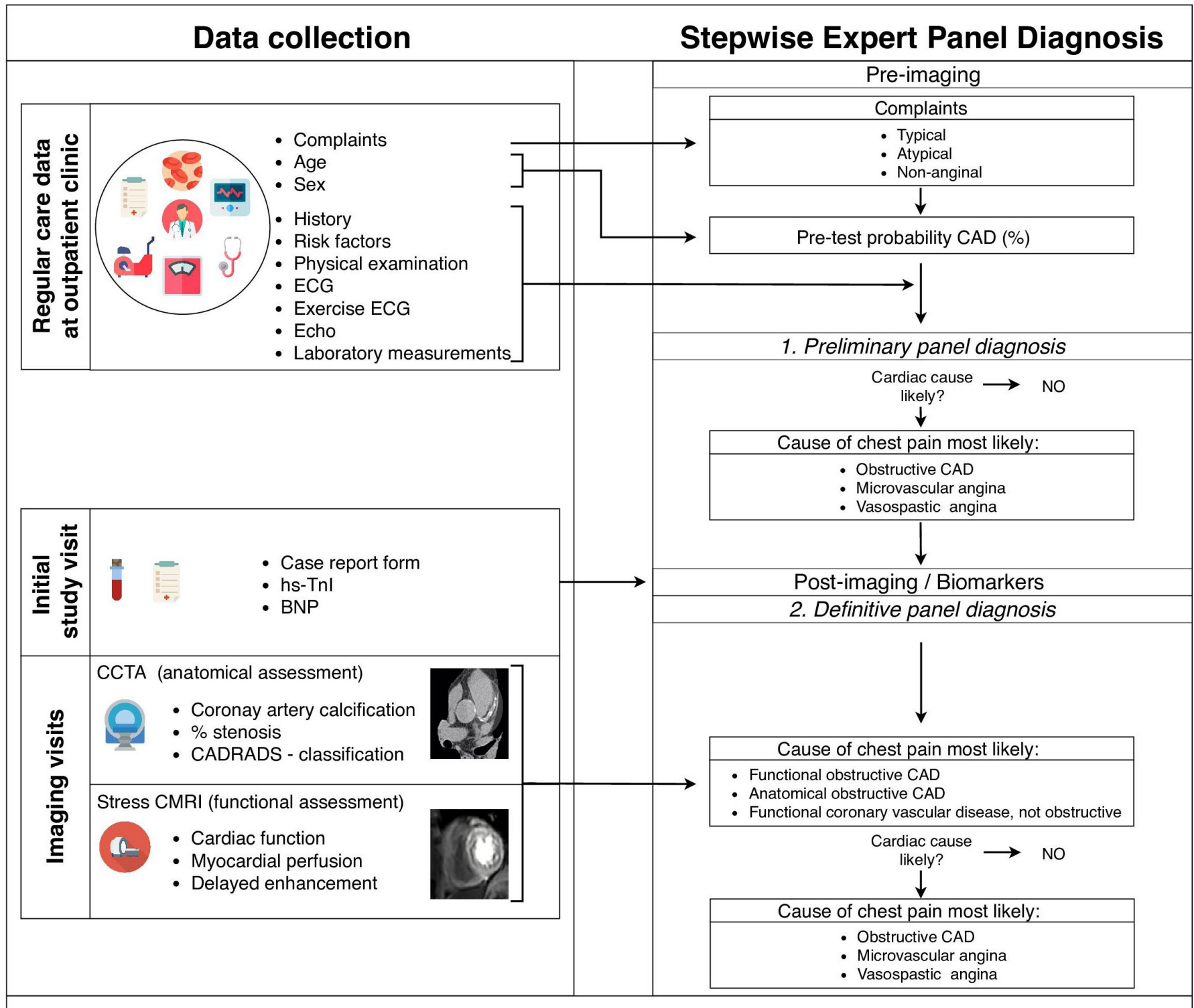

Figure 2 Workflow of data collection and implementation in the expert panel diagnosis. BNP, B-type natriuretic peptide; CAD, coronary artery disease; CADRADS, Coronary Artery Disease-Reporting and Data System; CCTA, cardiac CT angiography; $\mathrm{CMRI}$, cardiac MRI; hs-Tnl, high-sensitivity troponin-I.

variables, the outcomes of the CCTA and CMRI scans will be predicted in terms of the presence or absence of functional/anatomical coronary vascular disease. ARGUS will

\begin{tabular}{lcl}
\hline $\begin{array}{c}\text { Table } 1 \text { Classification of imaging results } \\
\text { Non-significant }\end{array}$ & Significant \\
\hline 1. Assessment of anatomical CAD on CCTA \\
Stenosis & $<50 \%$ & $\geq 50 \%$ \\
CADRADS classification & $0 / 1 / 2$ & $3 / 4 / 5$
\end{tabular}

2. Assessment of functional CAD on CMRI

\begin{tabular}{l} 
Myocardial ischaemia No Yes \\
\hline CAD, coronary artery disease; CADRADS, Coronary Artery \\
Disease-Reporting and Data System; CCTA, cardiac CT \\
angiography; CMRI, cardiac MRI.
\end{tabular}

function as the clinical validation study for the developed algorithm.

\section{STUDY STATUS}

ARGUS is actively recruiting participants. The number of participants recruited at the time of submission is 92 . Complete data of 70 participants are available, of whom $10.9 \%$ (5 women) were diagnosed with CMRI-detected functional coronary vascular disease and $16.4 \%$ (13 women) with CCTA-detected anatomical coronary vascular disease. The current inclusion rate (five participants per week) is sufficient to reach the goal of 400 included participants at the end of the inclusion period of 3 years.

\section{PATIENT AND PUBLIC INVOLVEMENT}

Participants from the outpatient clinic CCN were involved in the design of the study and evaluation of the study 
protocol. Participants receive information on the study at summary level during the enrolment and after the study will be completed and are asked to participate in evaluation of the study.

\section{ETHICS AND DISSEMINATION}

The study protocol was approved by the Institutional Review Board of the UMC Utrecht (NL_57077.041.16). The study is conducted according to the principles of the Declaration of Helsinki and in accordance with the Medical Research Involving Human Subjects Act. The study does not interfere with regular patient care, and all patients will be treated at the discretion of their cardiologist or general practitioner according to the appropriate guidelines. All participants give written informed consent for the study procedure. Results will be disseminated through national and international conferences and in peer-reviewed journals of cardiovascular disease.

\section{Author affiliations}

${ }^{1}$ Central Diagnostic Laboratory, University Medical Center Utrecht, Utrecht, the Netherlands

${ }^{2}$ Laboratory of Experimental Cardiology, University Medical Center Utrecht, Utrecht, the Netherlands

${ }^{3}$ Medical Imaging Systems BV, MEDIS, Leiden, the Netherlands

${ }^{4}$ Cardiology, Cardiology Centers Netherlands, Utrecht, the Netherlands

${ }^{5}$ Cardiology, HeartLife Clinics, Utrecht, the Netherlands

${ }^{6}$ Department of Epidemiology, Julius Center for Health Sciences and Primary Care, Utrecht, the Netherlands

${ }^{7}$ Radiology, University Medical Centre Utrecht, Utrecht University, Utrecht, the Netherlands

Acknowledgements We sincerely thank Ms Jonne Emanuel-Hos and Gideon Valstar for their outstanding efforts in and support to the HELPFul-ARGUS Study. We would like to thank Evelyn Velema, Merel Schurink and Marja Maat-Leersum for their contribution to the recruitment of participants and their aid in validating and processing the raw data. We also thank Danny Elbersen, Sander van de Weg Nanique Tulkens, Naomi Parr, Hemse Al-Khamisi, Lianne Granneman and Thuur Peeters for work-up of the biobank samples. We thank Suzan Kemper-Bongers and the UPOD team for extracting and combining the regular care data for analysis. We thank all people involved from HeartLife Klinieken Utrecht and the Cardiology Center of the Netherlands, specifically the team at Cardiology Center Utrecht. We sincerely thank all people involved from the radiology department of the UMC Utrecht for their efforts to perform both CCTA and CMRI in this study. A special thanks to all patients who participated in the study.

Contributors $\mathrm{FG}$ is the trial coordinator and responsible for collecting the data and undertook writing of the paper. ALME, SHB, A-Mv0 and LMO guided the writing of the paper. DK, GP, JHCR and DH were involved in the design and description of the study methods. RM, MEW and LH are involved in participant recruitment and made substantial improvements to the paper. NCO-M is the trial statistician who guided the design of the statistical analysis plan and made substantial improvements to the paper. $\mathrm{SH}, \mathrm{IH}, \mathrm{TL}$ and HMdR were involved in the methodological design, drafting of the study protocol and made substantial improvements to the paper. HMdR is the principal investigator who is the overall responsible for the design, conduct and decision to submit for publication. All authors have read and given final approval of the submitted manuscript.

Funding The original investigator-initiated project HELPFul received funding from the Dutch Heart Foundation 2013T084 (Queen of Hearts). The current ARGUS Study using CMRI and CCTA is supported by funding from CVON 2017-22 ARGUS.

Competing interests None declared.

Patient consent for publication Not required.

Provenance and peer review Not commissioned; externally peer reviewed.
Supplemental material This content has been supplied by the author(s). It has not been vetted by BMJ Publishing Group Limited (BMJ) and may not have been peer-reviewed. Any opinions or recommendations discussed are solely those of the author(s) and are not endorsed by BMJ. BMJ disclaims all liability and responsibility arising from any reliance placed on the content. Where the content includes any translated material, BMJ does not warrant the accuracy and reliability of the translations (including but not limited to local regulations, clinical guidelines, terminology, drug names and drug dosages), and is not responsible for any error and/or omissions arising from translation and adaptation or otherwise.

Open access This is an open access article distributed in accordance with the Creative Commons Attribution Non Commercial (CC BY-NC 4.0) license, which permits others to distribute, remix, adapt, build upon this work non-commercially, and license their derivative works on different terms, provided the original work is properly cited, appropriate credit is given, any changes made indicated, and the use is non-commercial. See: http://creativecommons.org/licenses/by-nc/4.0/.

\section{ORCID iDs}

Floor Groepenhoff http://orcid.org/0000-0002-1583-701X

Sophie Heleen Bots http://orcid.org/0000-0002-4483-5582

\section{REFERENCES}

1 Hoorweg BB, Willemsen RT, Cleef LE, et al. Frequency of chest pain in primary care, diagnostic tests performed and final diagnoses. Heart 2017;103:1727-32.

2 Conroy RM, Pyörälä K, Fitzgerald AP, et al. Estimation of ten-year risk of fatal cardiovascular disease in Europe: the score project. Eur Heart J 2003;24:987-1003.

3 Wilson PW, D'Agostino RB, Levy D, et al. Prediction of coronary heart disease using risk factor categories. Circulation 1998;97:1837-47.

4 Foldyna B, Udelson JE, Karády J, et al. Pretest probability for patients with suspected obstructive coronary artery disease: reevaluating Diamond-Forrester for the contemporary era and clinical implications: insights from the promise trial. Eur Heart $J$ Cardiovasc Imaging 2019;20:574-81.

5 Genders TSS, Steyerberg EW, Alkadhi $\mathrm{H}$, et al. A clinical prediction rule for the diagnosis of coronary artery disease: validation, updating, and extension. Eur Heart $J$ 2011;32:1316-30.

6 Truong QA, Rinehart S, Abbara S, et al. Coronary computed tomographic imaging in women: an expert consensus statement from the Society of cardiovascular computed tomography. $J$ Cardiovasc Comput Tomogr 2018;12:451-66.

7 Jespersen L, Hvelplund A, Abildstrøm SZ, et al. Stable angina pectoris with no obstructive coronary artery disease is associated with increased risks of major adverse cardiovascular events. Eur Heart J 2012;33:734-44.

8 Johnston N, Schenck-Gustafsson K, Lagerqvist B. Are we using cardiovascular medications and coronary angiography appropriately in men and women with chest pain? Eur Heart J 2011;32:1331-6.

9 Sharaf B, Wood T, Shaw L, et al. Adverse outcomes among women presenting with signs and symptoms of ischemia and NO obstructive coronary artery disease: findings from the National heart, lung, and blood Institute-sponsored women's ischemia syndrome evaluation (wise) angiographic core laboratory. Am Heart J 2013;166:134-41.

10 Sara JD, Widmer RJ, Matsuzawa Y, et al. Prevalence of coronary microvascular dysfunction among patients with chest pain and nonobstructive coronary artery disease. JACC Cardiovasc Interv 2015;8:1445-53.

11 Prevalence and prognosis of nonobstructive coronary artery disease in patients undergoing coronary angiography or coronary computed tomography angiography: a meta-analysis. Mayo Clinic Proceedings. Elsevier 2017.

12 Barr PR, Harrison W, Smyth D, et al. Myocardial infarction without obstructive coronary artery disease is not a benign condition (ANZACS-QI 10). Heart, Lung and Circulation 2018;27:165-74.

13 Shaw LJ, Bugiardini R, Merz CNB. Women and ischemic heart disease: evolving knowledge. J Am Coll Cardiol 2009;54:1561-75.

14 Camici PG, Crea F. Coronary microvascular dysfunction. N Engl J Med 2007;356:830-40.

15 Ong P, Safdar B, Seitz A, et al. Diagnosis of coronary microvascular dysfunction in the clinic. Cardiovasc Res 2020;116:841-55.

16 Mathew RC, Bourque JM, Salerno M, et al. Cardiovascular imaging techniques to assess microvascular dysfunction. JACC: Cardiovascular Imaging, 2020: 13. 1577-90.

17 Kramer CM, Barkhausen J, Bucciarelli-Ducci C, et al. Standardized cardiovascular magnetic resonance imaging (CMR) protocols: 
2020 update. Journal of Cardiovascular Magnetic Resonance 2020;22:1-18

18 Knuuti J, Ballo H, Juarez-Orozco LE, et al. The performance of non-invasive tests to rule-in and rule-out significant coronary artery stenosis in patients with stable angina: a meta-analysis focused on post-test disease probability. Eur Heart J 2018;39:3322-30.

19 Hoffmann U, Ferencik M, Udelson JE, et al. Prognostic value of noninvasive cardiovascular testing in patients with stable chest pain: insights from the promise trial (prospective multicenter imaging study for evaluation of chest pain). Circulation 2017;135:2320-32.

20 Valstar GB, Bots SH, Groepenhoff F, et al. Discovery of biomarkers for the presence and progression of left ventricular diastolic dysfunction and heart faiLure with preserved ejection fraction in patients at risk for cardiovascular disease: rationale and design of the helpful case-cohort study in a Dutch cardiology outpatient clinic. BMJ Open 2019;9:e028408.

21 Abbara S, Blanke P, Maroules CD, et al. SCCT guidelines for the performance and acquisition of coronary computed tomographic angiography: a report of the Society of cardiovascular computed tomography guidelines Committee: endorsed by the North American Society for cardiovascular imaging (NASCl). $J$ Cardiovasc Comput Tomogr 2016;10:435-49.

22 van der Bijl N, Joemai RMS, Geleijns J, et al. Assessment of Agatston coronary artery calcium score using contrast-enhanced $\mathrm{CT}$ coronary angiography. AJR Am J Roentgenol 2010;195:1299-305.
23 Cury RC, Abbara S, Achenbach S, et al. CAD-RADS(TM) Coronary Artery Disease - Reporting and Data System. An expert consensus document of the Society of Cardiovascular Computed Tomography (SCCT), the American College of Radiology (ACR) and the North American Society for Cardiovascular Imaging (NASCl). Endorsed by the American College of Cardiology. J Cardiovasc Comput Tomogr 2016;10:269-81.

24 Cerqueira MD, Weissman NJ, Dilsizian V, et al. Standardized myocardial segmentation and nomenclature for tomographic imaging of the heart. A statement for healthcare professionals from the cardiac imaging Committee of the Council on clinical cardiology of the American heart association. Circulation 2002;105:539-42.

25 Knuuti J, Wijns W, Saraste A, et al. 2019 ESC guidelines for the diagnosis and management of chronic coronary syndromes: the task force for the diagnosis and management of chronic coronary syndromes of the European Society of cardiology (ESC). Eur Heart $J$ 2020;41:407-77.

26 Sollie A, Roskam J, Sijmons RH, et al. Do GPs know their patients with cancer? Assessing the quality of cancer registration in Dutch primary care: a cross-sectional validation study. BMJ Open 2016;6:e012669.

27 Reitsma JB, Kardaun JW, Gevers E, et al. [Possibilities for anonymous follow-up studies of patients in Dutch national medical registrations using the Municipal Population Register: a pilot study] Ned Tijdschr Geneeskd 2003;147:2286-90. 